\title{
Automated Analysis of Multi Site MRI Phantom Data for the NIHPD Project
}

\author{
Luke Fu, Vladimir Fonov, Bruce Pike, Alan C. Evans, and D. Louis Collins \\ McConnell Brain Imaging center, Montreal Neurological Institute, \\ Quebec, Canada \\ lukelpfu@yahoo.com, louis.collins@mcgill.ca, \\ \{vfonov, bruce, alan\}@bic.mni.mcgill.ca \\ www.bic.mni.mcgill.ca/nihpd/
}

\begin{abstract}
In large multi-center studies it is important to quantify data variations due to differences between sites and over time. Here we investigate inter-site variability in signal to noise ratio (SNR), percent integral uniformity (PIU), width and height using the American College of Radiology (ACR) phantom scans from the NIHPD project. Longitudinal variations are also analyzed. All measurements are fully automated. Our results show that the mean SNR, PIU and the 2 metric values were statistically different across sites. The maximum mean difference in diameter across sites was $2 \mathrm{~mm}(1.1 \%)$, and the maximum mean difference in height was $2.5 \mathrm{~mm}(1.7 \%)$. Over time, an average drift of $0.4 \mathrm{~mm}$ per year was observed for the diameter while a drift of $0.5 \mathrm{~mm}$ per year was observed for the height. Trends observed over time often depended not only on site, but also on modality and scanner manufacturer.
\end{abstract}

\section{Introduction}

The National Institute of Health funded MRI study of normal brain development aims to collect MRI data from a representative sample of normal, healthy pediatric brains [5]. The purpose is twofold: 1) to provide the largest normative database to date of the developing human brain for comparison with children who have neurological, developmental and psychiatric disorders; and 2) provide longitudinal data for comparative studies involving brain maturation and behavioral/cognitive development in normal children. The study consists of approximately 546 children (aged 10 days to 18 years) who will make 3 visits over a period of 5 years, and involves 6 different data collection sites in the United States. In this paper, this normal pediatric database is termed NIHPD.

The study requires multi-site collaboration due to the quantitative and demographic nature of the project - over 500 children with varying financial backgrounds scanned across the United States over several years. All data collected are transferred to the Data Coordinating Center (DCC) in Montreal, where databasing, initial statistical analysis and image processing are carried out.

Since data received at the DCC originate from six different sites, inherent variability is expected within the acquired data [4]. Differences such as scanner make (Siemens/GE), model type and acquisition software all contribute to the overall 
differences between sites. These differences can cause complications in image processing: inherent difference in intensity uniformity can affect tissue classification [2], whereas difference in geometric distortion can affect the size of segmented structures [3]. Clearly, both artifacts will contribute to overall uncertainty in the estimation of cranial structures - hence, an unbiased, systematic characterization of inter-site variance is of great importance.

One possible solution to ensure quality control in a large multi-site MRI study is the use of standardized phantoms. The ACR phantom is the official MRI accreditation phantom, as proposed by the American College of Radiology [6]. The phantom consists of a plastic cylinder with inner dimensions of $190 \mathrm{~mm}$ (wide) by $148 \mathrm{~mm}$ (long), and is filled with a solution of $10 \mathrm{mM}$ nickel chloride and $75 \mathrm{mM}$ sodium chloride. It has several unique plastic structures embedded inside the cylinder, which is used to measure image qualities of a MRI scanner. The seven quantitative tests proposed by the ACR MRI accreditation program are: 1) Geometric accuracy, 2) high contrast spatial resolution, 3) slice thickness accuracy, 4) slice position accuracy, 5) image intensity uniformity, 6) percent signal ghosting and 7) low contrast object detectability. For the NIHPD study, data collecting sites are required to conduct ACR phantom scans at least once per month.

In this study we investigate the effects of inter-site scanner variability on geometric accuracy (height, width), signal to noise ratio (SNR) and percent integral uniformity (PIU) of 1048 phantom volumes acquired in the NIHPD project (visit 1), using an automated approach. Eventually, we hope to enhance the current technique to include all 7 of the ACR accreditation tests.

\section{Methods}

The following pediatric study centers (PSCs) are involved in the NIHPD data collection process: Boston Children's Hospital, Children's Hospital Medical Center of Cincinnati, Children's Hospital of Philadelphia, University of Texas Health Science Center at Houston, Washington University St. Louis and University of California Los Angeles. The scanners used to scan the ACR phantom across all sites include: Siemens Magnetom Vision, Siemens Magnetom Sonata, GE Excite Signa and GE Genesis Signa. Actual acquisition parameters depend on the scanner's manufacturer.

\subsection{Scanners}

For the GE scanners, the acquisition details are as follows:

- 3D T1 weighted scans: TR $=22 \mathrm{~ms}, \mathrm{TE}=10-11 \mathrm{~ms}$, Field of View $(\mathrm{FoV})=250$ $\mathrm{mm}$ Inferior-Superior (IS) x $250 \mathrm{~mm}$ Anterior Posterior (AP), 124 slices of $1.7 \mathrm{~mm}$ slice thickness;

- $\mathrm{T} 1$ weighted fallback scans: $\mathrm{TR}=500 \mathrm{~ms}, \mathrm{TE}=10 \mathrm{~ms}, \mathrm{FoV}=250 \mathrm{~mm}$ AP $\times 220$ mm Left-Right (LR), 66 slices of $3 \mathrm{~mm}$ thickness;

- 2D Proton Density weighted (PDW)/T2 weighted scans: TR = $3500 \mathrm{~ms}$, TE1 = 17 $\mathrm{ms}, \mathrm{TE} 2=119 \mathrm{~ms}, \mathrm{FoV}=250 \mathrm{~mm}$ AP $\times 220 \mathrm{~mm}$ LR, 88 slices of $2 \mathrm{~mm}$ thickness;

- 2D PDW/T2W fallback scans: TR $=3500 \mathrm{~ms}$, TE1 $=17 \mathrm{~ms}$, TE2 $=119 \mathrm{~ms}, \mathrm{FoV}=$ $250 \mathrm{~mm}$ AP x $190 \mathrm{~mm}$ LR, 30 - 60 slices of $3 \mathrm{~mm}$ thickness. 
The Siemens scanners had same parameters with slight modifications:

- 3D T1 weighted scans: TR $=25 \mathrm{~ms}, \mathrm{TW}=11 \mathrm{~ms}$, Field of View $($ FoV $)=256 \mathrm{~mm}$ Inferior-Superior (IS) x 256 mm Anterior Posterior (AP), $124-128$ slices of 1.7 mm slice thickness;

- $\mathrm{T} 1$ weighted fallback scans: TR $=500 \mathrm{~ms}, \mathrm{TE}=12 \mathrm{~ms}, \mathrm{FoV}=256 \mathrm{~mm}$ AP x 224 $\mathrm{mm}$ Left-Right (LR), 66 slices of $3 \mathrm{~mm}$ thickness;

- 2D Proton Density weighted $(\mathrm{PDW}) / \mathrm{T} 2$ weighted scans: $\mathrm{TR}=3500 \mathrm{~ms}$, TE1 = 17 $\mathrm{ms}, \mathrm{TE} 2=119 \mathrm{~ms}, \mathrm{FoV}=256 \mathrm{~mm}$ AP $\times 224 \mathrm{~mm}$ LR, 88 slices of $2 \mathrm{~mm}$ thickness;

- 2D PDW/T2W fallback scans: TR $=3500 \mathrm{~ms}$, TE1 $=17 \mathrm{~ms}$, TE2 $=119 \mathrm{~ms}, \mathrm{FoV}=$ $256 \mathrm{~mm}$ AP x $192 \mathrm{~mm}$ LR, 22 slices of $3 \mathrm{~mm}$ thickness.

PSCs were asked to perform scans using the parameters listed above for the test subjects as well as the ACR phantoms. Ideally, the ACR phantom should be placed in the scanner according to the recommendations of the ACR MRI accreditation protocol. The MRI data collected from the scanner console is to be transferred to a Study Workstation (SWS) using DICOM transfer, then from the SWS to the DCC using another DICOM transfer. The files are eventually transformed to MINC format [12] before the automated analysis is performed.

\subsection{Signal to Noise Ratio}

In our study the SNR was defined as:

$$
S N R=\frac{\text { Mean Phantom Signal }}{(0.8 \times \text { Mean Background Signal })}
$$

Since the background noise has a Raleighian distribution, the 0.8 factor is used to correct it for Gaussian characteristics of the MR signal [8]. The mean foreground signal, mean background signal and the background standard deviation are all estimated using a program available in the MNI-autoreg package called MINCSTATS. A bimodal-T threshold value for pixel intensity is calculated to separate foreground and background signal. The foreground and background regions are eroded to avoid voxels with partial volume effects.

\subsection{Percent Integral Uniformity}

The Percent Integral Uniformity is calculated using the formula:

$$
P I U=100 \times\left(1-\left[\frac{(\text { high }- \text { low })}{(\text { high }+ \text { low })}\right]\right)
$$

This is almost the same uniformity equation proposed by Colombo and Magnusson $[10,13]$. According to the original definitions of the ACR MRI accreditation manual, high/low denotes regions of high/low signal value in slice 7 of the ACR phantom, and should be $1 \mathrm{~cm}^{2}$ in area [6]. The calculations done in this study follow a slightly different approach - instead of analyzing by slice, parameters from the 
entire volume are evaluated. Using a histogram, the upper and lower 5 percentile of the entire phantom volume are calculated and used to define the "high" and "low" signal values in Equation 2.

\subsection{Geometric Uniformity}

The geometric accuracy test specified by the ACR MRI accreditation manual requires measuring the diameter of the mid slice of the ACR phantom in 4 different directions (i.e. 4 diameter measurements per volume). In this study the automated script measures two extra planes ( $60 \mathrm{~mm}$ above and below the center plane) in 6 different directions, generating a total of 18 diameter measurements per volume. The measurements are done using a bimodal threshold to delineate phantom volume signal (representing the boundary/edges of the phantom) and the background signal. Identified boundaries have their coordinates recorded, and the lengths are calculated as the difference between the top boundary coordinates and the bottom boundary coordinates. Height is also measured using the same method.

\section{Results}

Measurements obtained from the ACR analysis were examined using the JMP statistics package (JMPIN version 5.1.2).

\subsection{Inter-site Variability}

A oneway ANOVA by site was carried out for each of the 4 variables (SNR, PIU, diameter, height) followed by Tukey-Kramer's Honestly Significant Difference (HSD) test. While T1, T2, PD and their associated fallbacks were acquired and analyzed, only results for T1 data are reported (Fig. 1) due to space limitations in this paper.

The SNR was found to have a mean of 111.8 with an average standard error of 4.2 across the 8 sites. The observed SNR values were significantly different between different sites $(\mathrm{F}$ ratio $=39.6633, \mathrm{p}<0.0001)$, and the maximum difference was found to be 78. The Tukey-Kramer HSD shows that sites 5 and 6 have mean SNR significantly different than the other 5 sites.

In terms of PIU, data from the 7 sites showed less variance than the SNR - the mean PIU value was found to be $83.6 \%$ with an average standard error of $0.6 \%$. The F ratio value of $9.4064(\mathrm{p}<0.0001)$ confirms that the difference in means from each site is statistically significant, and the largest difference between the mean PIU values was $5.6 \%$. Tukey-Kramer HSD results show that only site 6 had a PIU that is significantly different to the other sites.

The average phantom diameter was found to have a mean of $189.6 \mathrm{~mm}$ and an average standard error of $0.1 \mathrm{~mm}$. The differences between the mean phantom diameters of different sites were less than $1.9 \mathrm{~mm}(1 \%)$, but the differences were still statistically significant $(\mathrm{F}$ ratio $=48.0775, \mathrm{p}<0.0001)$. From Tukey-Kramer HSD it was found that sites 2 and 5 had significantly different means than the rest of the sites. 



Fig. 1. Plot of SNR, PIU, phantom diameter and height vs. site for the T1 modality. The horizontal line represent the grand mean of all sites, while the diamond represent $95 \%$ normal confidence interval centered at the sample mean.

The average phantom height was determined to be $148.4 \mathrm{~mm}$, with an average standard error of $0.1 \mathrm{~mm}$. The difference between the largest group mean and the smallest was $1.9 \mathrm{~mm}(1.3 \%)$, and the difference in mean height across the 7 sites was also statistically significant ( $\mathrm{F}$ ratio of 57.0644, $\mathrm{p}<0.0001$ ). Tukey-Kramer HSD revealed that the different mean heights can be grouped roughly into 4 pairs, where the mean height difference was statistically insignificant within groups.

\subsection{Longitudinal Variability}

The results presented here represent changes in PIU, SNR, phantom diameter/height in time (Fig. 2). An average was determined for the 6 modalities in each site, with statistically significant trends in time detected and analyzed. Here we report results for site 2 and site 7 only due to space limitations.

\subsubsection{Site 2}

PIU averages across different modalities from site 2 had a mean value of $84.2 \%$. The averages were within $1 \%$ of each other, and no statistically significant trends were associated with any of the modalities. Similar to site 1 data, SNR for the site 2 also showed great range - from the mean T1 value of 115.5 to the mean T2 value of 36.6. No statistically significant trends were observed except for the T1 data, which showed a significant decreasing trend (SNR decrease of 10.8 per year) with time.

The 2 metrics (diameter and height) were found to have a mean value of $189.0 \mathrm{~mm}$ and $147.5 \mathrm{~mm}$, respectively. Five out of six modalities showed an increasing diameter in time (T1WF, T2, T2WF, PD, PDWF, mean increase of $0.5 \mathrm{~mm}$ per year), while the height remained constant for all modalities. 

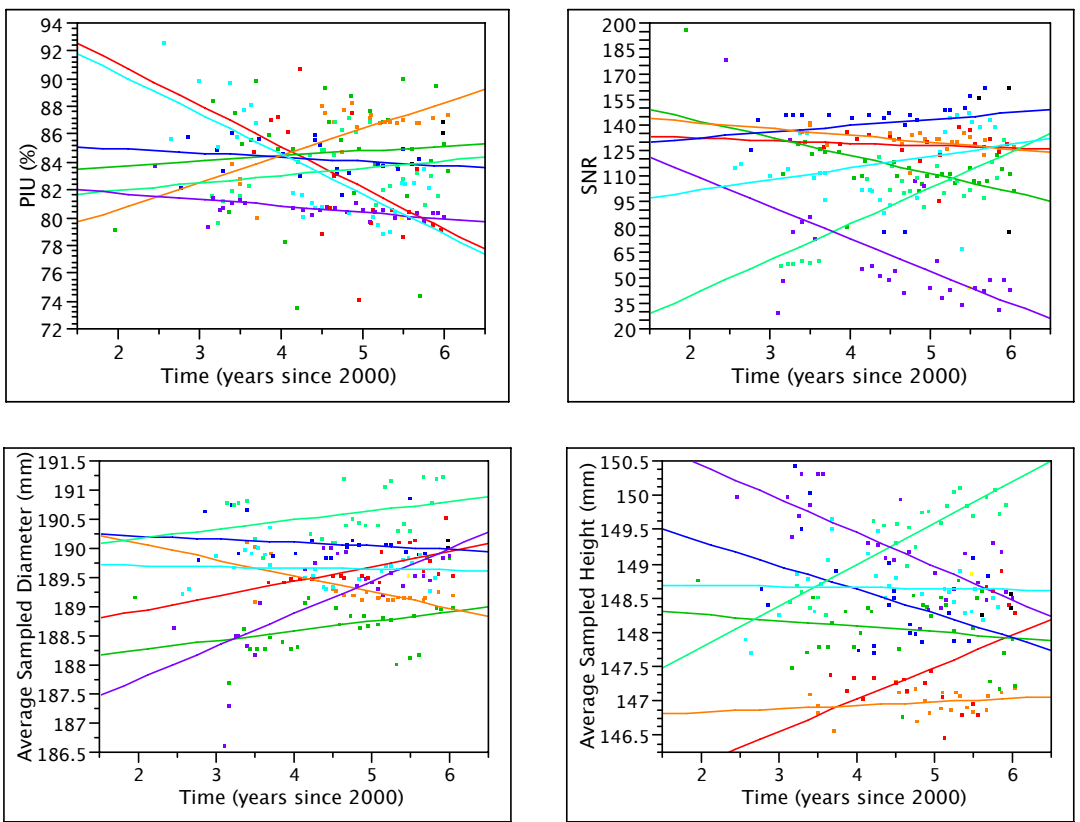

Fig. 2. Longitudinal T1 plots of PIU, SNR, diameter and height for all sites. The legend is as follows: Red $=$ Site 1 , Green $=$ Site 2 , Blue $=$ Site 3, Orange $=$ Site 4 , Lime $=$ Site 5, Purple $=$ Site 6 , Aqua $=$ Site 7 .

\subsubsection{Site 7}

For Site 7, the average PIU was calculated to be 79.3\%: however, none of the 6 modalities analyzed showed significant trends in time. Analysis of the mean SNR values reported a maximum of 116.6 for the $\mathrm{T} 1$, and a minimum of 28.1 for the T2. T1 also showed significant increase with time, while T1WF and T2WF showed significant decrease with time.

Analysis of the metrics found the average phantom diameter to be $189.7 \mathrm{~mm}$. Trends in time for the diameter showed erratic behavior, with T2 increasing in time but T1WF, PD and PDWF decreasing in time. The average change (in modalities that showed significant changes in time) was $0.3 \mathrm{~mm}$ per year. The average phantom height was determined to be $148.7 \mathrm{~mm}$, and all modalities showed a significant average decrease of $0.4 \mathrm{~mm}$ per year.

\section{Discussion}

The aim of this paper is to quantify inter-site variability of a multi-center MRI study using the ACR phantom. The parameters used to characterize variability are SNR, PIU and geometric accuracy. The 3 parameters were processed automatically for all 1048 phantom data. 
While all data went through a manual QC process before integration into the database, inclusion of some lower quality data in the analysis may affect the overall statistics - e.g., streak(s)/bands, data cropped near the edges (resulting in overlapping images) and data with high noise/significant ghosting. Data considered to be of lower quality are believed to be less than $2 \%$ of the entire ACR phantom dataset.

Bourel et al. reported for their Vision scanner excellent stability in terms of SNR and uniformity over 3 years [9]. In our study, SNR and PIU both showed small yet statistically significant trends in time, depending on manufacturer, modality and site. In terms of metrics, results obtained showed a slight drift over time but are otherwise stable - this was in agreement with findings from Ewers and Schnack $[11,14]$. The largest change per year was a decrease of $2.1 \mathrm{~mm}$ in diameter from Site 5 PDWF, which corresponds to a change of $1.1 \%$ in length over time - the majority of trends are considerably smaller in magnitude, with an average of $0.4 \mathrm{~mm}$ shift per year in diameter $(0.2 \%)$ and $0.5 \mathrm{~mm}$ shift per year for height $(0.3 \%)$. This is likely due to drifts in the gradient coil strengths in time, with an apparent difference in magnitude between the $\mathrm{x} / \mathrm{y}$ plane and the $\mathrm{z}$ plane causing the geometric distortions $[1,7]$.

To the author's knowledge, a multicenter QA study involving over one thousand ACR MRI phantom images has not been reported in previous literature. The results obtained in this study should serve as a useful reference for future multicenter studies.

\section{Conclusion}

Inter-site variability is an important factor to consider in large multi-site studies. In this paper we investigated the effect of site and time versus SNR, PIU, ACR phantom diameter and height for the NIHPD project. Across sites, the means were found to be statistically different from the grand mean for all 4 parameters. Longitudinally, the presence of statistically significant trends depended on the site involved as well as modality used in the scan.

\section{References}

1. Mah D., Steckner M., Palacio E., Mitra R., Richardson T., Hanks G.E.: Characteristics and quality assurance of a dedicated open $0.23 \mathrm{~T}$ MRI for radiation therapy simulation. Medical Physics, Vol. 29(11):2541-2547, November 2002

2. Jones R.W., Witte R.F.: Signal intensity artifacts in clinical MR imaging. Radiographics Vol. 20(3):893-901, May-June 2000.

3. Lemieux L., Barker G.J.: Measurement of small inter-scan fluctuations in voxel dimensions in magnetic resonance images using registration. Medical Physics, Vol. 25(6):10491054, June 1998.

4. Jovicich J., Czanner S., Greve D., Haley E., Kouwe A., Gollub R., Kennedy D., Schmitt F., Brown G., MacFall J., Fischl B., Dale A., Reliability in multi-site structural MRI studies: Effects of a gradient non-linearity correction on phantom and human data. NeuroImage, Vol. 30(2):436-43, April 2006.

5. Evans A.C., Brain Development Cooperative Group: The NIH MRI study of normal brain development. NeuroImage, Vol. 30(1):184-202, March 2006.

6. American College of Radiology: Phantom Test Guidance for the ACR MRI Accreditation Program. American College of Radiology, September 2000. 
7. Chen C.C., Wan Y.L., Wai Y.Y., Liu H.L.: Quality Assurance of Clinical MRI Scanners Using ACR MRI Phantom: Preliminary Results. Journal of Digital Imaging, Vol. 17(4):279-284, December 2004.

8. Haacke E.M., Brown R.W., Thompson M.R., Vankatesan R: Magnetic Resonance Imaging - Physical Principles and Sequence Design. Wiley-Liss, $1^{\text {st }}$ Edition, June 1999.

9. Bourel P., Gibon D., Coste E., Daanen V., Rousseau J.: Automatic quality assessment protocol for MRI equipment. Medical Physics, Vol. 26(12), December 1999:2693-2700.

10. Colombo P., Baldassarri A., Del Corona M., Mascaro L., Strocchi S.: Multicenter trial for the set-up of aMRI quality assurance programme. Magentic Resonance Imaging, Vol. 22:93-101, 2004.

11. Ewers M., Teipel S.J., Dietrich O., Schonberg S.O., Jessen F., Heun R., Scheltens P., van de Pol L., Freymann N.R., Moeller H.J., Hampel H.: Multicenter assessment of reliability of cranial MRI. Neurobiology of Aging, epub. [Vol. 27(8):1051-9, August 2006].

12. Neelin P.: The MINC File Format: From Bytes to Brains. NeuroImage, Vol. 7(4), 1998.

13. Magnusson P., Olson L. E.: Image analysis methods for assessing levels of image plane nonuniformity and stochastic noise in a magnetic resonance image of a homogeneous phantom. Medical Physics, Vol. 27(8):1980-1994, August 2000.

14. Schnack H.G., van Haren N. E. M., Hulshoff Pol H. E., Picchioni M., Weisbrod M., Sauer H., Cannon T., Huttunen M., Murray R., Kahn R. S.: Reliability of Brain Volumes from Multicenter MRI Acquisition: A Calibration Study. Human Brain Mapping, Vol. 22:312320,2004 . 